\title{
Inhalt des Heftes 5
}

HEMPEL, K.-J., und TREFF, W. M., Die Gliazelldichte bei klinisch Gesunden und
Schizophrenen . . . . . . . . . . . . . . . . . . . . . . . . . . 371
TREFF, W. M., und HEMPEL, K.-J., Quantitative Untersuchungen über relative
Zell- und Zellkernvolumina bei klinisch Gesunden und Schizophrenen . . . . 411

Das „Journal für Hirnforschung" wird - wie bis 1942 das „Journal für Psychologie und Neurologie" — die Forschungsergebnisse des Institutes für Hirnforschung und allgemeine Biologie in Neustadt/Schwarzwald veröffentlichen. Im Mittelpunkt der Forschungen dieses Institutes steht die Hirnanatomie, und zwar jene Teile derselben, die die wichtigsten Erkenntnisquellen für die räumlichen Beziehungen zwischen materiellem Hirngeschehen und $\mathrm{Be}-$ wußtseinserscheinungen darstellen. Vertiefung der architektonischen Gliederung des Gehirns, Aufdeckung des anatomischen Ausdrucks individueller Besonderheiten Gesunder, Kranker und „zurechnungsfähiger“ Asozialer, Ausnutzung der pathologischen Anatomie für die Schaffung einer ätiologischen Klassifikation der sogenannten funktionellen Neurosen und Psychosen, Klärung der aufbauenden und reparatorischen Funktionen des metamitotischen Arbeitskernes der Nervenzellen: das sind gegenwärtig die Hauptforschungsgebiete des Institutes.

\section{Bestellungen an eine Buchhandlung erbeten}

Wenn Sie unsere Literatur nicht in Ihrer Buchhandlung erhalten können oder Schwierigkeiten bei der Beschaffung haben, dann wenden Sie sich bitte an eine der nachstehenden Auslieferungsstellen oder direkt an den Verlag.

\section{Auslieferung für die Deutsche Demokratische Republik:} LKG Leipziger Kommissions- und Großbuchhandel Leipzig C1, Leninstraße 16

Auslieferung für die Bundesrepublik:

Kunst und Wissen, Erich Bieber, Stuttgart S, Wilhelmstr. 4-6

Auslieferung für das gesamte Ausland:

Deutscher Buch-Export und -Import, GmbH, Leipzig C 1, Postschließfach 276

Akademie-Verlag,

Berlin W 1, Leipziger Str. 3-4, Ruf: 220441 Sammelnummer Telegrammadresse: Akademieverlag Berlin

\footnotetext{
Herausgeber und verantwortlich für den Inhalt: Dr. Cécile und Prof. Oskar Vogt, Institut für Hirnforschung und allgemeine Biologie, Neustadt/Schwarzwald. Verlag: A kademie-Verlag GmbH., Berlin W 1, Leipziger Str. 3-4 (Fernruf: 2204 41); Postscheckkonto: Berlin 35021 . Bestellnummer dieses Heftes: 1018/4/5. Das „Journal für Hirnforschung"c erscheint in 2 wanglosen Heften von verschiedenem Umfang. 6 Hefte bilden einen Band. Preis je Einzelheft 12,- DM. Ein Band 72,- DM. Satz und Druck: VEB Druckhaus „Maxim Gorki“, Altenburg. Veröffentlicht unter der Lizenznummer ZLN 5029 des Ministeriums für Kultur, Hauptverwaltung Verlagswesen. Printed in Germany.
} 